\title{
Study on Uropathogens with Antimicrobial Susceptibility Pattern in the Elderly at a Tertiary Care Hospital- Mangadu, South Chennai India \\ $\underline{\text { (Original Article) }}$
}

Authors

\section{Dhevahi Elumalei ${ }^{1}$, Sumathi.G. ${ }^{1}$ Sambandan A.P ${ }^{1}$, Natrajan.V.S ${ }^{2}$, Dinakaran. ${ }^{3}$}

${ }^{1}$ Department of Microbiology Sri Muthukumaran Medical College Hospital and Research Institute,

${ }^{2}$ Medical Superidendent \& Department Head ENT Sri Muthukumaran Medical College Hospital and Research Institute

${ }^{3}$ Department of Gastroentrologist \& Department of Geriatric Melmaruvathur Medical College

Corresponding Author

Dr Dhevahi Elumalei

Assistant Professor, Department of Microbiology, Sri Muthukumaran Medical College \& Research Institute. 17/20, Siraj street, Ayyavoo Naidu Colony, Ammainthakarai, Chennai, 600029.9840520088

Email: deviranga@gmail.com

\section{Abstract}

Urinary tract infections (UTI) second most common cause of infectious disease among 65 yr older the elderly population (>65years) ${ }^{1}$. Usually over diagnosed, treated on the basis of nonspecific clinical signs and symptoms. Elderly diagnosis always require the presence of new urinary symptoms, with or without systemic symptoms.

Aim: Our study aims to describe the uropahogens, antimicrobial resistance rates among elderly patients attending our hospital near Mangadu South of Chennai.

Study Subjects \& Results: A total of 468 urine specimens were analysed from elderly patients above sixty years of age. 343 were culture positive. Antibiotic susceptibility pattern of these patients were analysed. Our study shows among the GNB the E.Coli as the commonest, followed by Klebsiella. Staphylococus aureus was seen in many of the elderly population among the cocci group. Amikacin, Levofloxacin, Gentamycin and Nitrofurantoin were effective for treatment of UTI. Evidence showed resistance to Ampicilliin, Cotrimoxazole, cephalosporins.

Conclusion: study was able to standardize the pattern of antibiotic treatment within the elderly group after the analysis of antibiogram sensitivity.

Keywords: urinary tract infection, elderly, Bacterial agents, Antimicrobial susceptibility

\section{Introduction}

Urinary tract infections (UTI) are second most common infectious complaints among the geriatric clinics. UTI becomes a factor in the decline and death among geriatric patients $\mathbf{4 , 6 , 7 , 1 1}$ although this has not been fully investigated at a clinical or scientific level. Spectrum of UTI ranges from symptomatic bacteriuria to bacteremic infection. UTI with bacteremia has a high mortality in the older population, with studies reporting a 28 day mortality of $5 \% .^{1}$ Usually in elderly UTI is commonly over 
diagnosed and treated on the basis of nonspecific clinical signs and symptom, with vague presentations incontinence and a change in mental status, or fatigue as the only symptoms. ${ }^{\underline{2}}$ Diagnosis are complicated by the fact that many elderly people have preexisting incontinence or dementia. Antimicrobial resistance is on the rise and a cause of major concern in many countries ${ }^{7}$ There are several risk factors for colonisation, infection and spreading of antibiotic resistant bacteria among elderly. Extensive antibiotic prescription is related to a higher prevalence of antibiotic resistant bacteria. The elderly are prescribed antibiotics more frequently than younger adults. Antimicrobial resistance studies in uropathogens among elderly residents however, there are considerable differences in resistance rates between countries ${ }^{2}$. UTI in the older patient requires the presence of various new urinary symptoms, with or without systemic symptoms for diagnosis along with use of narrow spectrum antimicrobial agents. Local guidelines on antibiogram will always reflect local resistance patterns and available agents. Guidelines need to be updated to reflect changes in the pattern of antimicrobial prescriptions to move from broad to narrow spectrum antimicrobials.Paucity of evidence-based guidelines for the management of UTI specifically in an elderly population in our society. An updated estimate of the native prevalence of antimicrobial resistance in uropathogens among the community will help in treatment of the elderly in Critical emergency situation. Based on these facts the study was prospectively designed and analysed.

\section{AIM \& OBJECTIVES}

This study is aimed to analyse the uropathogens in elderly patients with their pattern of antimicrobial resistance to determine and standardise the antibiotic treatment within the elderly group attending our hospital near Mangadu, South of Chennai.

\section{Materials and Methods}

A prospective analysis on urine culture specimens from elderly patients age (> 60 years) attending the outpatients and inpatient units of Sri Muthukumaran Medical College Hospital and Research Institute near Mangadu , South of Chennai formed the study subjects . A total of 468 single, mid-stream, urine specimen was collected from August 2012- April 2015. Clinical Details was noted, urine culture was done and analysed in the Microbiology Section. Dermographic data on the type of bacterial isolates from the urine specimens and antibiotic susceptibility patterns were recorded and tabulated.

\section{Methods of Isolation and identification:}

Urine specimen was inoculated onto nutrient, blood and MacConkey agar plates by streaking method. Inoculated plates were incubated aerobically at $37^{\circ} \mathrm{C}$ for 24 hours. Identification of pure isolates was done by observing morphological, cultural and biochemical characters ${ }^{1}$. "A culture with growth of potentially pathogenic bacteria was normally considered positive if the number of colony forming units per liter $(\mathrm{CFU} / \mathrm{mL})$ was $\mathrm{fg} 10^{5}$. Antibiotic sensitivity testing was performed using the Kirby-Bauer disc diffusion method according to the Clinical and Laboratory Standards Institute Guidelines 2 Multi-discs diffusion were done using ten different antibiotics. Antimicrobial drug susceptibility testing for Amikacin $A K, 30 \mu \mathrm{g}$, Nitrofurantoin NIT $(300 \mu \mathrm{g})$ Cotrimoxazole $C O T, 25 \mu \mathrm{g}$, Norfloxacin $N X$, $(10 \mu \mathrm{g})$, Ampicillin $A M P, 10 \mu \mathrm{g}$, Cefotaxime $30 \mu \mathrm{g}$, Cefoxitin $C X$, Nalidixic acid $N A$ - Gentamicin $G, 10 \mu \mathrm{g}$, Ceftazidime (30mg), Ciprofloxacin CIP, $(5 \mu \mathrm{g})$, and Imipenum ( IMP) was done on all bacterial isolates. Interpretation of results was done based on the diameter of the zone. 


\section{Results}

Table I-Shows Culture Positivity among the Male and Female Patients in Elderly Total no of samples analysed $n=468$

\begin{tabular}{|l|l|l|l|}
\hline Sex & Samples & $\%$ freq & $\begin{array}{l}\text { Nos. of positive } \\
\text { cultures }\end{array}$ \\
\hline Male & 198 & 42.3 & $125(63.1 \%)$ \\
\hline Female & 270 & 57.6 & $218(80.7 \%)$ \\
\hline
\end{tabular}

In our study overall culture positivity in the elderly is $73.2 \%$, male patients $63.1 \%$ positivity, while in females patients $80.7 \%$ of cases.

Table II: Shows the Age Break up between Male and Female Elderly Subjects

\begin{tabular}{|l|l|l|}
\hline & Age & Growth \\
\hline \multirow{2}{*}{$\begin{array}{l}\text { Male } \\
(\mathrm{n}-198) 42.3 \%\end{array}$} & 65-75yrs & $98 \quad(49.5 \%)$ \\
\cline { 2 - 3 } & 76-85 years & $18 \quad(9 \%)$ \\
\cline { 2 - 3 } & $86-95$ years & $9 \quad(4.5 \%)$ \\
\hline \multirow{2}{*}{ Total } & $125 \quad(63.1 \%)$ \\
\hline \multirow{2}{*}{$\begin{array}{l}\text { Female } \\
\text { 57-270) }\end{array}$} & 65-75 years & $172 \quad(63 \%)$ \\
\cline { 2 - 3 } & 76-85 years & $38 \quad(14.1 \%)$ \\
\cline { 2 - 3 } Total & $86-95$ years & $8 \quad(3 \%)$ \\
\hline
\end{tabular}

Our study showed Female population between sixty to seventy years to have a higher rate of infection $(63 \%)$.whereas males were found to be positive above 76 years.

Table-III-Shows Uropathogens isolated in our Elderly Population ( $\mathrm{n}=343)$

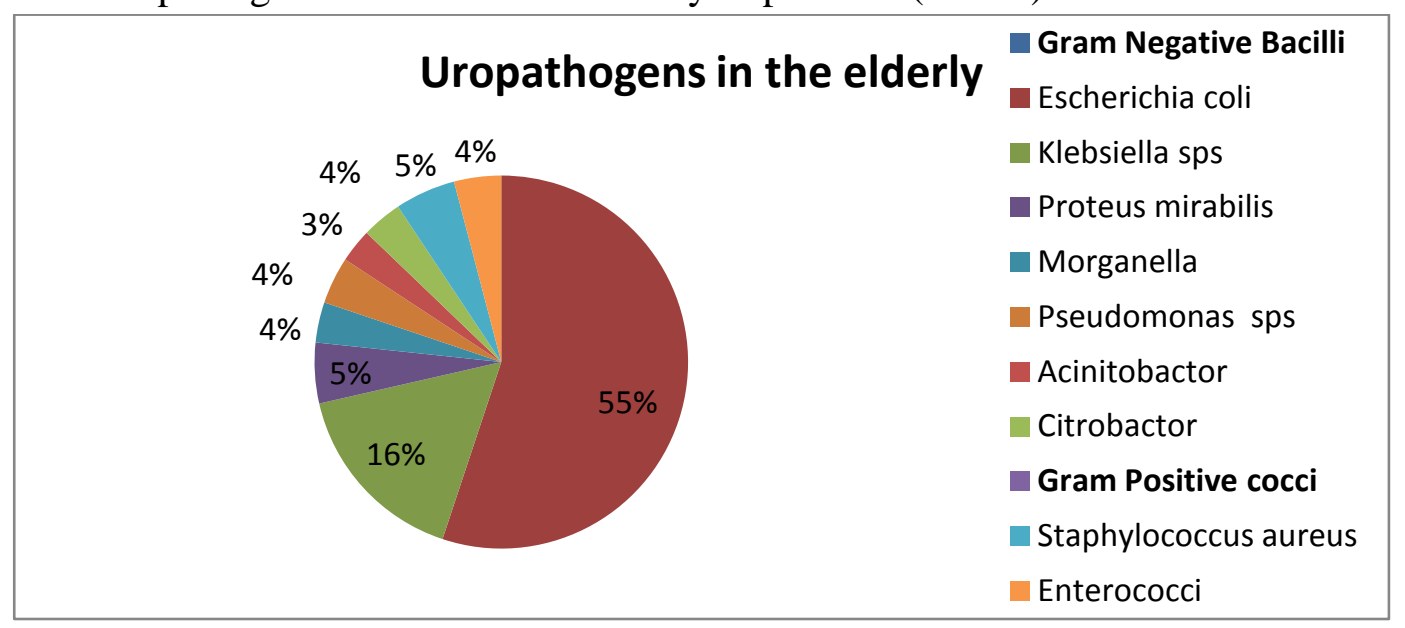

Among the Gram Negative bacilli E.Coli (55.1\%) was the highly observed pathogen next to klebsiella (16.3\%) sps. Staphylococcus aureus ( $5.2 \%$ ) was found to be common pathogen among the gram positive cocci in our study population. 
Table IV-Shows the Antibiogram Sensitivity Pattern among the Elderly group

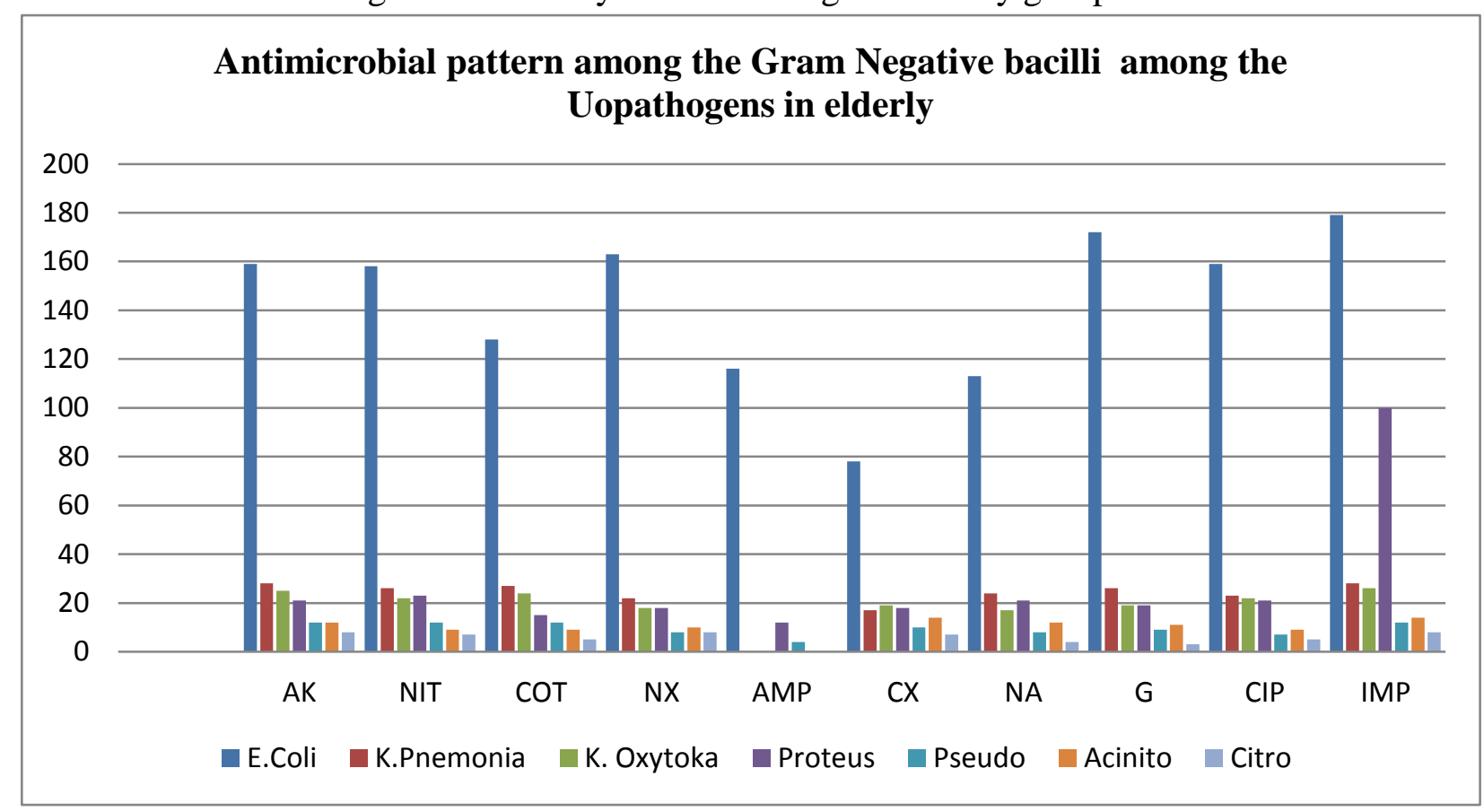

Amikacin (AK), Nitrofurantoin(NIT),Co-trimoxazole(COT), Norfloxacin(NX), Ampicillin(AMP), Cefoxitin(CX), Nalidixic acid(NA), Gentamycin(GEN), Ciprofloxacin( CIP), Imipenum(IMP)

The antibiogram pattern showed only 8 ESBL strains among the E.Coli and three Plasmid mediated AmpC production. There was no carbapenemases isolated or PDR or MBL strains among the elderly in our study. Ampicillin showed more of resistance among the uropathogens and was not suggested as the routine drug for the elderly.

\section{Discussion}

The diagnosis and management of UTI among elderly persons challenging to the clinician. Geriatric patients with UTI frequently have an atypical clinical presentation, an increased risk of drug-drug and disease-disease interactions. Therefore, iidentifying the etiological agent with its effective antibiotic sensitivity to the concerned UTI becomes a major key role in effective early management among elderly worldwide. Since the pattern of antimicrobial resistance varies in different regions and different categories of people with different age groups, our study was designed to evaluate a valuable data on uropathogens and their antimicrobial sensitivity pattern among elderly in our local population which will help and improve in the efficient empirical treatment. This study was confined to UTIs in elderly above the age of 65 years, and analysed the relationships between sex, isolated bacterial agents and antibiotic resistance of UTIs in our elderly population. Our study shows prevalence of UTI in the elderly to be $73.2 \%$. Our study correlated well with other studies similar to those reported from many other centers. ${ }^{6,7,9}$ Females had a higher rate of culture positivity $80 \%$ than males 63\%. (Table -I). The sex distribution of patients in our study is consistent with those of other reported studies, showing a statistically predominance of females with UTI (80\% of the positive cultures).The elevated incidence of infection among females is related to differences between the male, female genitourinary systems in anatomy and microflora 12.

Among the Uropathogens analysed, Enterobacteriaceae family were the most common 
microorganism isolated accounting $71.4 \%$ of total isolated bacteria and amongst them E. coli was the most predominant bacteria. (Table-2). There are earlier studies in agreement to present finding 12,13 In our study E.Coli (55.1\%) was the predominant pathogen followed by klebsiella (16.3\%) sps among the gram negative organisms. Our study correlated well with many other studies by ${ }^{\mathbf{1 2 , 1 3}}$ and Staphylococcus aureus ( 5.2\%) was found to be common pathogen among the gram positive cocci followed by Enterococci. (Table 3). Similarities and differences in the type, distribution of uropathogens may result from different environmental conditions in host factors according to each country. The prevalence of Gram-positive cocci was not high in our study.

Studies on the local guidelines for prescribing antimicrobials, based on resistant pattern are limited. Although our patient size is not very large, our study forms first of its kind among our local population, a pilot one which demonstrates the need for usage of narrow spectrum antibiotics after the culture reports, where we could prevent the development of resistant strain among the uropathogens.

In the present study a high level of bacterial resistance was seen to ampicillin and cephalosporin's against all the uropathogens isolated. This is similar to previous studies in the United States ${ }^{\mathbf{7 , 1 1}}$.Our study shows greater susceptibility to Amikacin and Nitrofurantoin, Cotimoxasole, Ciproflox, similar studies are reported. ${ }^{14}$ The high prevalence of resistance to the commonly used antibiotics such as ampicillin, cephalosporins has caused considerable alarm which is similar to shyamala et al. ${ }^{12}$. Based on the results of this study, it was revealed that the susceptibility of bacteria to ciprofloxacin and other antibiotics was similar to many studies ${ }^{14}$. Our study also generates the view to follow guidelines from other established countries like UK which advocates that trimethoprim should be used as the first-line antibiotic in complicated symptomatic UTI.
Nitrofurantoin is an alternative, although it should not be used in those with renal impairment. ${ }^{10}$ European guidelines are now moving towards fosfomycin trometamol or nitrofurantoin as firstand second-line agents with trimethoprim or cotrimoxazole advised only in areas where resistance rates for $E$. coli are less than $20 \% .^{8}$. Guidelines from Infectious Disease Society of America also published to follow Trimethoprimsulfamethoxazole as first-line agent for acute symptomatic lower urinary tract infection 3 days. Trimethoprim and ofloxacin were proposed as suitable alternatives. Fluoroquinolones were not recommended as firstline therapy unless there was a high level $(10 \%-20 \%)$ of trimethoprim or trimethoprim-sulfamethoxazole resistance. Nitrofurantoin and fosfomycin use may be more prominent if increasing resistance emerged. Recent, revised guidelines place nitrofurantoin as one of the first-line agents for UTI. Recent data suggests that nitrofurantoin can be safely administered to patients with creatinine clearance of at least $40 \mathrm{~mL} / \mathrm{min} / 1.73 \mathrm{~m}$ Nitrofurantoin achieves very low plasma concentrations, $40 \%$ of it is excreted in the urine, it maintains very low rates of resistance after 60 years of use, it is cost effective. For more highly resistant bacterial isolates, fosfomycin may be effective for older adults. It is more expensive than other oral agents. Vancomycin-resistant enterococci (VRE), methicillin-resistant $S$ aureus (MRSA), and extended-spectrum $\quad \beta$-lactamase $\quad$ (ESBL)producing gram-negative rods are usually susceptible to fosfomycin, and although the bacterial efficacy is lower than other first-line agents, it is an appealing oral outpatient alternative for resistant isolates. Fifteen randomized controlled studies concluded that short course antibiotics of 3-6 days could be adequate for treating uncomplicated UTI in older women. A need for more studies looking at optimum duration of narrow spectrum agents was highlighted. Another study in Scotland shows that Complicated UTIs, including upper UTI and 
bacteremic UTI, are treated with intravenous amoxicillin and gentamicin. Co-trimoxazole is used as an alternative in penicillin allergic patients in conjunction with gentamicin. According to our results, the efficacy of amikacin was comparable to other reports. The susceptibilitry pattern to the antibiotics for geriatric patients in UTI vary from place to place and from time to time.

Problems with antimicrobial resistance can also be tackled with more judicious use of antibiotics. Research has sought to uncover the ways of reducing antimicrobial prescribing in the older population. $^{12,13,14}$

\section{Summary}

Geriatric patients with UTI frequently have uncomplicated atypical clinical presentation, therefore around $40 \%$ of elderly population are incorrectly diagnosed, antibiotics are started empirically before the laboratory results of urine culture are available for UTI infections ${ }^{3}$. To ensure appropriate therapy, correct knowledge of the organisms that cause UTI and their antibiotic susceptibility is mandatory ${ }^{2}$ Our study certainly revels the common pathogen as E.Coli, along with the antibiogram pattern in our local population, thereby reducing inappropriate usage of antibiotics for asymptomatic bacteriuria. Our study is first of its kind in Mangadu, located in south of Chennai. The need for additional research on UTI among the elderly is imperative. There are currently very few studies determining the appropriate dosing and course duration for antimicrobial therapy. With emerging knowledge on antibiotic resistance and health care-associated infection, guidelines need to be updated to reflect the need to prescribe narrow spectrum agents when available and avoid empirical use of broad spectrum antibiotics.

\section{Conclusion}

Studies on local guidelines for prescribing antimicrobials based on resistant pattern are not much available. Although our patient size is not very large, our study is a pilot one which demonstrates the need for usage of narrow spectrum antibiotics after the culture reports, where we could prevent the development of resistant strain among the uropathogens in our community.

\section{Acknowledgements}

Author wishes to thank all the Microbiology department staff and technicians for their kind support.

\section{References}

1. Urinary tract infection in old age: overdiagnosed and over-treated. Age Ageing. 2000;29:297-298.

2. Woodford HJ, George J. Diagnosis and management of urinary tract infection in hospitalized older people. J Am Geriatr Soc. 2009;57: 107-114.

3. Optimal management of urinary tract infections in older people Louise A Beveridge, Peter $\mathrm{G}$ Davey, et.al., Interv Aging. 2011; 6: 173-180

4. Urinary Tract Infections in Elderly Persons. Chapter 32: Manisha JuthaniMehta et.al Department of Internal Medicine, Section of Infectious Diseases, Yale University School of Medicine, New Haven, Connecticut

5. The Incidence of uropathogens and their resistance pattern in a tertiary care hospital R. Shyamala and Janardhan Rao Der Pharmacia Lettre, 2013, 5 (1):316-317

6. Urinary Tract Infections in Older Women: A Clinical Review Lona Mody et al.JAMA. 2014 February 26; 311(8): 844854. doi:10.1001/jama.2014.303.

7. Infectious disease hospitalizations among older adults in the United States from 1990 through 2002.Curns AT, Holman RC, Sejvar JJ, Owings MF, Schonberger LB, Arch Intern Med 165: 2514-2520, 2005 
8. Urinary Tract infection in old age: Over diagnosed and over treated -Commentary. Age and Aging 2000; 29;297- 298

9. Urinary Tract Infections in Older Women: A Clinical Review Lona Mody et al.JAMA. February 26; 311(8): 844-854.

10. Optimal management of urinary tract infections in older people Louise A Beveridge, Peter G Davey, et.al., Interv Aging. 2011; 6: 173-180.

11. Antimicrobial resistance in urinary pathogens among Swedish nursing home residents remains low: a cross-sectional study comparing antimicrobial resistance from 2003 to 2012 Sundvall et al. BMC Geriatrics 2014, 14:30

12. The Incidence of uropathogens and their resistance pattern in a tertiary care hospital R. Shyamala and Janardhan Rao Der Pharmacia Lettre, 2013, 5 (1):316-317

13. Urinary Tract Infection at the Age Extremes: Pediatrics and Geriatrics Linda M. Dairiki Shortliffe, et., al. July 8, 2002 The American J Med. Volume 113 (1A) $57 \mathrm{~S}$

14. Antimicrobial resistance in urinary pathogens among Swedish nursing home residents remains low: a cross-sectional study comparing antimicrobial resistance from 2003 to 2012 Sundvall et al. BMC Geriatrics 2014, 14:30 